\title{
Optimal set-up of industrial scheduling software
}

\author{
D. Talbi*o, L. Geneste ${ }^{\circ}$, B. Grabot ${ }^{\circ}$, R. Prévitali*, Pascal Hostachy* \\ *Ortems S.A., Le Sextant - 150 Grande Rue de St Clair, F-69731 Caluire et Cuire Cedex- \\ France, \\ Email \{d.talbi, r.previtali, p.hostachy\}@ortems.fr \\ ${ }^{\circ}$ LGP-ENIT, 47, Avenue d'Azereix - BP 1629, F-65016 Tarbes Cedex - France, \\ Email: \{laurent, bernard\}@enit.fr
}

\begin{abstract}
For a long time, workshop managers have only expected from industrial schedulers to find quickly and sometimes interactively a feasible schedule. However, since the schedule governs the manufacturing resource usage, companies now require to optimize their schedule according to more or less complex criteria. This optimization is not only necessary in order to allow the company to remain competitive, but also in order to allow it to efficiently participate to Supply Chains in a Collaborative context. Many parameters are present in nowadays industrial schedulers which have a direct influence on the schedule quality. Tuning these parameters is a long and complex task if performed manually, which can be addressed by combinatorial optimization methods. Meta-heuristics have also been successfully used on specific scheduling problems, but the size of industrial problems and the necessity to provide a generic solving framework set specific difficulties. We show in this communication how several meta-heuristics can be integrated and combined in an environment allowing both to define a complex objective function, on the base of several elemental criteria, and to optimize the schedule by finding the best tuning of several tens of scheduling parameters. Typical results based on the cases of real workshops are provided.
\end{abstract}

\section{Keywords}

Scheduling, optimization, meta-heuristics.

\section{INTRODUCTION}

The introduction of micro-computers at the workshop level of the companies, some twenty years ago, has made possible the development of industrial scheduling software that were to be directly used by the workshop managers. During some years, obtaining quickly a feasible schedule for "classical" workshops in a userfriendly environment was considered as fully satisfactory but the increasing competitive pressure and the context of Supply Chains have progressively lead the workshop managers to be much more demanding. Since the schedules governs the manufacturing resource usage, finding a feasible schedule is nowadays not anymore sufficient and an emergent issue can be noticed in manufacturing companies concerning the optimization of their schedule, regarding various criteria. This 
internal optimization is nowadays a key factor in order to efficiently participate to Supply Chains, under condition that the chosen objective function not only expresses the internal objectives of the company, but also the global performance criteria of the Supply Chain.

In order to address this problem, a first idea could be to build the schedule using an optimization method. This can be done when building a dedicated scheduler from scratch, but it is not anymore possible when an existing industrial scheduler is used:

a The use of optimization methods sets modelling constraints which are usually poorly consistent with the huge set of technological constraints which have to be taken into account in order to be sure that the schedule is feasible [Farhoodi 1990, Smith 1992].

- The kernel of the industrial schedulers would need to be completely redeveloped, which would cost a lot with a very problematic profitability.

The challenge is so to optimize the result of a scheduling process which does not use an optimal method, i.e. to find the optimal set-up of the numerous parameters which influence the "quality" of the schedule. The problem is that the scheduling parameters often have mutual-influences; moreover, the effect of a parameter value on the schedule quality heavily depends on the characteristics of the scheduling problem (number of resources, types of routings, dispersion of processing times, average load, etc.) (see for instance Sim et al. 1994 and Pierreval \& Mebarki 1997). In that context, finding manually an "optimal", or near-optimal set-up orders remains a trial and error process, requiring both time and experience.

Combinatorial methods could be used in that purpose, and meta-heuristics like genetic algorithms, simulated annealing or Tabu search have among others been successfully used on specific scheduling problems (see for instance the review of [Jain \& Meeran 1998]). Nevertheless, the size of industrial problems combined with the necessity to provide a generic framework sets specific difficulties.

We describe here how various meta-heuristics can be integrated in an environment allowing both to define a complex objective function, on the base of several elementary criteria, and to optimize the schedule by finding the best tuning of the scheduling parameters. A prototype of this environment, called "Optimizer" has been developed and successfully tested in order to optimize the result of an industrial scheduler called Ortems@ $($, European market leader. The result of the optimization process on two real databases of users of the Ortems scheduler is shown.

\section{META-HEURISTICS AND SCHEDULE OPTIMIZATION: A PANORAMA}

Schedule optimization is in many cases a very complex problem since it is often NP-hard in the strong sense [Jain \& Meeran 1998]). As a consequence, Operation Research optimization techniques (such as Mixed Integer Linear Programming or Branch and Bound) are difficult to use on real size industrial problems with an acceptable processing time.

Other classical approaches to scheduling, like the use of priority dispatching 
rules (SPT-Shortest Processing Time, Slack Time...), provide only very poor performance according to an optimization context since the selection of an appropriate dispatching rule is difficult [Pierreval \& Mebarki 1997]. This explains the interest for the application of meta-heuristics to the problem of schedule optimization. Indeed, many research applications of meta-heuristics have shown the potential interest of these techniques for highly combinatorial problems [Corne $e t$ al. 1999]. Meta-heuristic approaches can be divided into three classes:

1. Local search techniques (such as Tabu Search [Glover \& Laguna 1993] and Simulated Annealing [Ingber 1993]) are based on the definition of a neighborhood of the current solution. A guided search in this neighborhood allows then to choose an appropriate neighbor for next step,

2. Evolutionary and bio-based algorithms (Genetic Algorithms [Alander 1995], Memetic Algorithms [Moscato 1989], Scatter Search [Glover 1998], Ant Colony Optimization [Dorigo \& Di Caro 1999]) use a number of solvers that build a population which improve through time its ability to solve the problems.

3. Mixed meta-heuristics (such as Ants Colony Optimization and Simulated Annealing, or Tabu Search and Genetic Algorithms) aim at improving single meta-heuristics by combining the advantages of several methods on a given optimization problem.

All these techniques have been used in the field of schedule optimization (see for instance [Dell'Amico \& Trubian 1993], [Van Laarhooven et al. 1992], [Duvivier et al. 1996], [Colorni et al. 1993] for job-shop scheduling). However, even if they show good properties in terms of optimization (especially for Tabu Search according to [Jain \& Meeran 1998]) they remain difficult to tune. Moreover, they generally aim at solving formal scheduling problems, with a limited number of parameters and rather simple objective functions (compared to industrial size scheduling problems). Consequently, the company Ortems S.A., editor of the Ortems scheduler, has decided two years ago to launch a study aiming at developing an environment for assessing the feasibility of using meta-heuristic approaches for the optimization of full-size scheduling problems, using the Ortems scheduler.

\section{SCHEDULE OPTIMIIZATION OF INDUSTRIAL SIZE}

In addition to the size of the scheduling problem itself (number of machines, products, operations, etc.), two points characterize a problem of industrial size in scheduling: the number of performance criteria allowing to build and objective function, some of them being possibly linked to other enterprises in a collaborative context, and the number of parameters which have an influence on the final result.

The manufacturing process is driven by multiple objectives, possibly conflicting ("decrease work in progress" and "increase service to the customer" for instance). The relevant objectives, and as a consequence the performance criteria allowing to express them, depend on the characteristics of the workshop, of the products which are manufactured and of the type of relation between the company and its customer in a Supply Chain Management context. Ortems includes a list of pre-determined "classical" performance indicators which application can be customized by the user 
(e.g. the user may specify on which horizon of time, on which product, on which machines a criteria is applied, according to its nature). Among others, criteria such as the utilization ratio, idle time or sum of set-up times can be considered for machines, while criteria such as the cycle time, lateness or idle time can be considered for orders. Fifteen basic performance indicators are basically provided, usually leading to several tens of criteria after customization by the user. New performance criteria can also be directly defined by the user, on the base of the dynamic information on the machines, orders or customer's situation available in the database. The way these criteria can be combined in order to allow the user to build his own objective function is described in next section.

Even if Ortems does not use a standard list-scheduling approach, some of the used scheduling parameters-or criteria-aim at selecting from a set of operations the one which shall be processed first on a resource. These parameters are e.g. sorting rules such as priority to the operation having the shortest processing time, the smallest slack time, the smallest sum of remaining processing times, etc. Other criteria allow to choose a machine among others when an operation can be performed by several machines: selection of the machine having the lowest load, of the machine with the longest waiting time, etc. Around sixty criteria are available in Ortems.

It has often be verified since [Montazeri \& Van Wassenhove 1990] that these types of parameters are rather "specialized": sorting orders or machines according to a single parameter usually leads to a high satisfaction of one criteria, and a poor satisfaction of the others. In a collaborative context, the objectives of a workshop manager are multiple and partially conflicting, since some of the objectives express the requirements of other partners of the Supply Chain. In that context, it is reasonable and effective (see for instance [Grabot \& Geneste 1994]) to sort machines and orders according to a compromise between several criteria. The optimization process therefore consists in finding the set of criteria used for a given schedule along with their relative importance in the compromise. The way the scheduling parameters are combined is described in the next section.

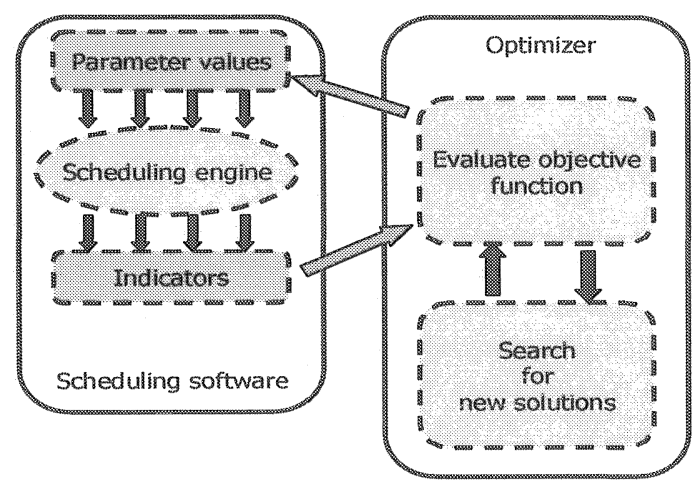

Figure 1 - Black box optimization 


\section{OPTIMIZER: A TOOL FOR SCHEDULE OPTIMIZATION}

\section{Basic principles}

As stated above, the aim of the study is to optimize the set-up of the parameters used by the scheduler. Of course, the performance provided by a set of values is only known after a schedule has been performed. As a consequence, we have defined a general purpose optimization environment based on a "black box" approach, a separation being made between search (performed by the Optimizer) and evaluation (performed by the Ortems scheduler) of solutions (see Figure 1). The evaluation of each solution is done by:

1. sending the parameter values as inputs to the scheduling software,

2. running a schedule,

3. retrieving indicator values as outputs of the scheduling software then

4. computing the objective function value. On the basis of this evaluation, and considering also the past evaluations, the search procedure decides upon a new set of input values.

\section{Development issues}

The optimizer environment was implemented in Java and integrated into the Ortems e-SCM product (i.e. the scheduler). Communication between the optimizer and the scheduling engine is made via APIs, XML and CORBA. The construction of new dedicated performance indicators can be done using the Ortems Profiler, which is a e-SCM component allowing the user to define his own indicators using each data contained in the data base.

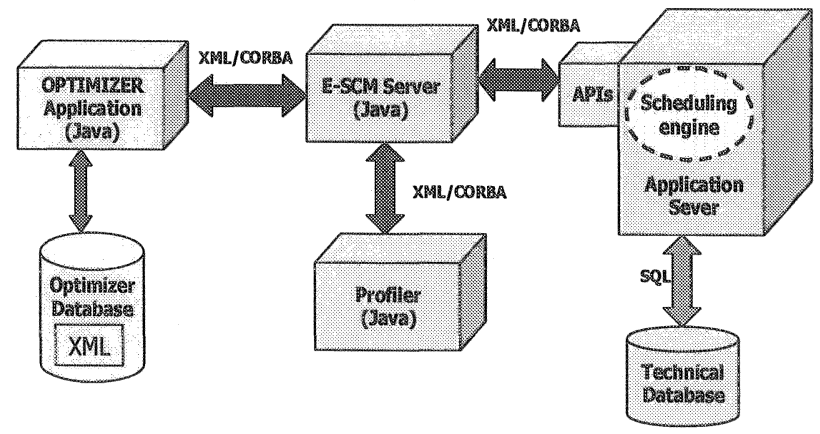

Figure 2 - The Architecture

Several scheduling techniques can be selected in the scheduler (forward scheduling, backward scheduling, etc.) which have an influence on the parameters to set-up. All the data regarding the system to optimize are stored in an XML database. This database is also used to store solutions saved by users, with their corresponding configurations (optimization method, objective function weights, etc.). This architecture enables to distribute the processing of the optimization algorithms on 
several computers, allowing the computing time to be drastically reduced. Several views and tables are available, allowing the user to follow the evolution of the objective function and indicator curves all along the optimization process (see Figure 3). The design of an objective function and the optimization process are described in the next sections.

\section{Description of an objective function}

Usually, it is not sufficient to have a single objective for the optimization of a manufacturing system, especially in a collaborative context. A set of objectives is therefore needed [Weigert et $a l$. 2000]. In order to aggregate the different objectives, we use an additive weighting method: scalar values given by adding weighted values of objectives determine the quality of solutions. The advantage of this method is its very simple management. A problem is that using a weighted sum requires to use normalized values, although minimum and maximum values are not a priori known by the manager. In order to solve this problem, we use a normalization according to an a-priori "goal" value expressing the final objective of the manager regarding this criterion. If our problem is to minimize the objective functions $f_{1}, f_{2}, \ldots, f_{n}$; the aggregated function can be written as:

$$
f(x)=\sum p_{i}\left(\frac{f_{i}(x)-\delta}{\delta_{i}-\delta}\right)
$$

where $p_{i}$ is the weight associated to the $\mathrm{i}^{\text {th }}$ objective, $\delta=-1+\min _{i} \delta_{i}$, and $\delta_{i}$ is an a priori goal value for the $\mathrm{i}^{\text {th }}$ objective. $\delta$ is used to translate the values of $f_{i}$ and $\delta_{i}$, in order to avoid the cases where they can take negative or null values.

\section{Use of the parameters in the scheduling process}

The standard version of Ortems uses a quasi-ordered lexicographical approach [Pomerol and Barbara-Romero 1993] in order to apply the scheduling parameters for solving a conflict between operations or machines. I.e. a scheduling criterion, like the Shortest Processing Time rule, the Slack Time rule etc., selects a set of operations in conflict, among which a second parameter will be applied in order to select a shorter set. The process is repeated until only a single operation is selected. Since this method does not allow making compromises, it has been replaced by a weighted sum in our study, setting again normalization problems.

Let $\mathrm{E}$ be a set of $\mathrm{n}$ conflicting operations (or machines) and a set of $\mathrm{m}$ scheduling parameters which can be used to solve the conflict. We can select the best operation according to a given parameter i by optimizing a numerical function $\mathrm{C}_{\mathrm{i}}$ defined on $\mathrm{E}$. 


$$
\bar{C}_{i}=\frac{C_{i}-\min C_{i}}{\max C_{i}-\min C_{i}}
$$

The conflict is solved by optimizing the aggregated function: $\Sigma w_{i} \bar{C}_{i}$, where $w_{\mathrm{i}}$, $i=1, \ldots, m$, are weights varying between 0 and 1 , and $\bar{C}_{i}$ is the normalized value of $C_{i}$. The normalization method used is: if $C_{i}$ is to be minimized and if $C_{i}$ is to be maximized.

Our problem is then to find the values of weights which allow to achieve good scheduling results.

$$
\overline{C_{i}}=\frac{\max C_{i}-C_{i}}{\max C_{i}-\min C_{i}}
$$

\section{Choice of the optimization methods}

As stated in section 4.1, the Optimizer is a Black Box optimization system, which evaluates the quality of a solution after performing a schedule. The time needed to achieve this schedule can vary from few seconds to hours according to the complexity of the industrial manufacturing process. Therefore, choosing the right heuristic is very important consideration in complex cases, since a "good" solution should be found in few steps.

As a first test, we implemented three of the simplest local search heuristics: Random Sampling (RS), multistart Random Hill Climbing (RHC) and Simulated Annealing (SA) algorithms. The first two heuristics move from a solution to another by selecting the first neighbor improving the objective function value, whereas SA can accept a move that worsen the objective function.

In RS algorithm, neighboring solutions are obtained by making a uniform random change in the values of all parameters. Unlike RS, the multistart RHC and SA algorithms make a change on only one parameter, chosen at random. SA does not perform well when the computation time allotted is short in comparison with the complexity of the problem [Phan and al. 2002(a)] [Phan and al. 2002(b)], which is the case in most of real applications we encountered. In such situations, our goal is to obtain a "good" solution as soon as possible and a local optimum can be acceptable. A simple RHC starting from the best known solution shows to be more efficient in such situations. When we do not have starting values, we can also use RS for few iterations, and use the best solution found to start Hill Climbing.

A specific neighborhood generation function was used to change parameters in RHC and SA algorithms. This function is based on a modification of LEO algorithm for simulation optimization [Barretto et al. 1999]. The LEO algorithm (Linear move and Exchange move Optimization algorithm) makes use of two procedures for neighborhood generation. The first procedure (linear move) increases or decreases a variable according to a given value. The second (exchange move) changes its value for another value generated by a uniform distribution between the minimum and the maximum values. We added a third procedure which eliminates a parameter if the quality of the solution is not decreased. We shall see in the following sections that 
these rather simple techniques can be efficiently used for our optimization problem.

\section{Choice of a parameter during the optimization process}

Considering the important number of available parameters (more than thirty!), a critical choice in order to insure a fast convergence of the method is to choose the parameter having the greatest influence at the right moment. Several strategies are now under evaluation, including a pre-selection using the manager expertise. The first tests have been performed by choosing a parameter at random, making an important change in its value, then perform a schedule. If the result does not change, the parameter weight is set to zero. Otherwise, it is included in the list of active parameters. Nevertheless, the possible correlation between parameters asks many interesting questions which are now under consideration.

\section{FIRST RESULTS}

All the tests were conducted on an $800 \mathrm{MHz}$ Pentium IIII computer, with $1 \mathrm{~GB}$ of total RAM memory.

\section{Example 1}

The Optimizer was firstly tested in order to optimize the schedule of a company producing fragrance and flavors. The manufacturing system consists in 136 machines and the considered production planning included 1329 jobs with many complex scheduling constraints (synchronous jobs, tank machines, ... etc.). This company was using the Ortems software with specific parameters values, obtained thanks to a considerable experience in the use of the scheduler. The objectives were to minimize the makespan and the mean work order delays (WOD1, WOD2, WOD3) for three groups of work orders with different importance degrees. The aggregated objective function was written as follows:

$$
\text { MAKESPAN/240 + (WOD1 - 10)/20 + } 0.8 *(\text { WOD2 - 10)/10 + } 0.5 *(\text { WOD3 - 10) }
$$

A first a priori selection allowed to consider that 43 parameters were to be tuned. Figure 3 shows the evolution of the objective function after two hours of calculation. The first point was calculated using the initial situation of the real manufacturing system. The method used is a Random Sampling during the first ten iterations. The best solution is then used to start a Hill Climbing search. At each hill climbing iteration, a parameter is chosen at random and its value is changed, then a scheduling of the real manufacturing system is done. The results obtained are summarized in table 1 . 


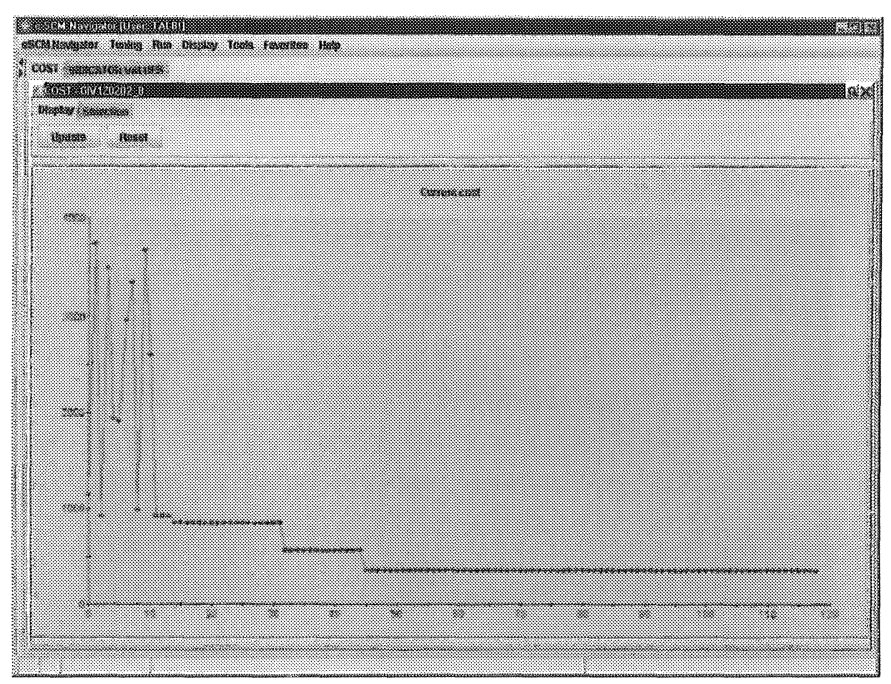

Figure 3 - Objective function evolution for the current solution

These results can still be improved if we restart from other points in the search space. The quality of the final result usually results from a compromise between the available time and the required quality of the solution: we are so close to an "anytime" problematic.

Table 1 - Indicator values before and after optimization

\begin{tabular}{|c|c|c|}
\hline Indicator & Before & After \\
\hline WOD1 & 62 days & 37 days \\
\hline WOD2 & 70 days & 35 days \\
\hline WOD3 & 14 days & 6 days \\
\hline MAKESPAN & 402 days & 371 days \\
\hline
\end{tabular}

\section{Example 2}

The second test was performed on a company working in aerospace subcontracting. Its manufacturing system is composed 152 machines, and 8556 orders were to be scheduled. A first analysis led to 53 parameters to be tuned. The objective function is to maximize the "ratio service", defined as the ratio between the number of early orders and the total number of orders. Figure 4 shows that the "ratio service" increased from $33 \%$ to $38 \%$ after about 3 hours of computation, although the initial performance has been obtained by experienced users and consultants.

\section{CONCLUSIONS}

From an industrial point of view, an important consideration is that tuning the parameters of a scheduling software is often a difficult and therefore expensive task. 
A significant progress can be achieved if using appropriate automated algorithm to support this task. Another important issue is that the problem of tuning parameters is not limited to the scheduling domain, and Optimizer is expected to provide a convenient and powerful tool in a wide range of application fields, including the optimization of several correlated schedules along a Supply Chain.

As it can be seen, many a priori choices have been made in order to allow quickly the development of an environment implementing the method. In spite of this, the results have been spectacular: on real customer cases, improvements of the performance criteria have always been observed in comparison with the best set-up found by the customer after several months of use of the scheduler.

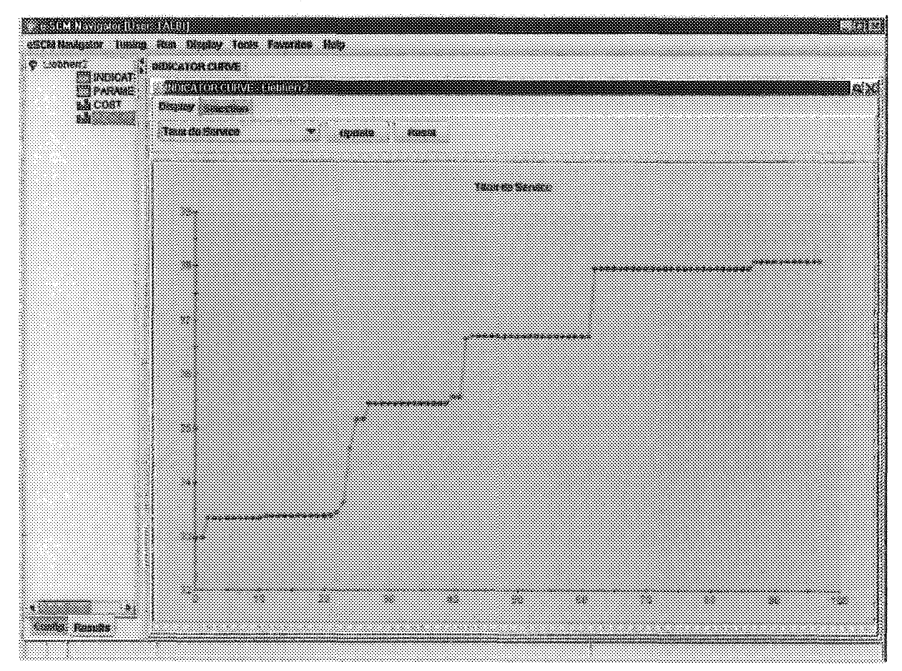

Figure 4 - Evolution of the Ratio Service

Many directions of research are now possible in order to still improve these results.

- A first direction of improvement is in the parameter reduction procedure, which reduces the size of the problem and allows to intensify the search in promising regions. We think that a good optimization algorithm cannot use a parameter reduction procedure without allowing parameters to be reintroduced later in the optimization process. We can also make use of statistical procedures based on visited solutions and /or on past experiments to guide the search in order to reduce the number of objective function evaluations. The robustness of the solution proposed is also very important in many practical situations, where a solution cannot be revised easily. Another idea is to relax some constraints during the optimization process, then to re-introduce these constraints and find a solution in the neighborhood of the optimal solution found with relaxed constraints.

m Another direction concerns the normalization of objective functions, since the method we used appears to be too dependent on the "goal" values specified by the user. Some authors [Weigert and al. 2000] proposed another approach, which dynamically normalizes the values of objective functions by using the 
knowledge on the visited solutions.

Nevertheless, we do think that this study shows the feasibility and interest of a meta-heuristic combinatorial optimization approach not only for solving limited research scheduling problems, but as a part of widely used market products.

\section{REIERENCES}

[1] Alander J. T., An Indexed Bibliography of Genetic Algorithms in Manufacturing, Lance Chambers ed., Practical Handbook of Genetic Algorithms: New Frontiers, Volume II, CRC Press, 1995.

[2] Barretto, M. R. P., Eldabi, T., Chwif, L., Paul, R. J., Simulation optimization with the linear move and exchange move optimization algorithm, Proceedings of the 1999 Winter Simulation Conference, P. A. Farrington, H. B. Nembhard, D. T. Sturrock, and G. W. Evans Eds, 1999.

[3] Colorni A., Dorigo M., Maniezzo V., and Trubian M., Ant system for Job-shop scheduling, Belgian Journal of Operations Research, Statistics and Computer Science, vol. 34, n'1, pp $39-54,1993$.

[4] Corne D., Dorigo M. and Glover F., New Ideas in Optimization, McGraw-Hill, 1999.

[5] Dell'Amico A. M., Trubian A. M., Applying Tabu Search to the Job-shop Scheduling Problem, Annals of Operation Research, 41, 1993.

[6] Dorigo M., Di Caro G., The Ant Colony Optimization Meta-Heuristic, in D. Corne, M. Dorigo and F. Glover Eds, New Ideas in Optimization, McGraw-Hill, pp. 11 - 32, 1999.

[7] Duvivier D., Preux Ph., and Talbi E-G., Genetic algorithms applied to the job-shop scheduling problem, in FUCAM'96, Workshop on Production Planning and Control, Mons, Belgique, September 1996.

[8] Farhoodi, F., A knowledge-based approach to dynamic job-shop scheduling, International Journal of Computer Integrated Manufacturing, vol. 2, n³, pp. 84 - 95, 1990.

[9] Glover F. and Laguna M., Tabu search, in Modern Heuristic Techniques for Combinatorial Problems, C. Reeves ed., Blackwell, Oxford, UK, 1993.

[10] Glover, $\mathbb{F}$., A Template for Scatter Search and Path Relinking, in Artificial Evolution, Lecture Notes in Computer Science 1363, J.-K. Hao, E. Lutton, E. Ronald, $\mathbb{M}$. Schoenauer and D. Snyers Eds., Springer, pp. 13 - 54, 1998.

[11] Grabot B., Geneste L., Dispatching rules in scheduling: a fuzzy approach, International Journal of Production Research, vol. 32, n4, pp. 903 - 915, 1994.

[12] Ingber L., Simulated annealing: Practice versus theory, Journal of Mathematical and Computer Modelling, vol. 18, n¹1, pp $22-59,1993$.

[13] Jain A. S. and Meeran S., A state-of-the-art review of job-shop scheduling techniques, Technical report, Department of Applied Physics, Electronic and Mechanical Engineering, University of Dundee, Dundee, Scotland, 1998.

[14] Montazeri M., Van Wassenhove L.N., Analysis of dispatching rules for an FMS, International Journal of Production Research, vol. 28, $n^{\circ} 4$, pp. $785-802,1990$.

[15] Moscato P., On Evolution, Search, Optimization, Genetic Algorithms and Martial Arts: Towards Memetic Algorithms, Caltech Concurrent Computation Program, C3P Report 826, 1989.

[16] Phan, V., Skiena, S., Sumazin, P., (a) A model for analysing Black Box Optimization, Extended Abstract, citeseer.nj.nec.com/phan02model.html, 2002. 
[17] Phan, V., Sumazin, P., Skiena, S., (b) A time-sensitive system for black-box combinatorial optimization, proceedings of workshop on Algorithm Engineering and experimentation ALENEX '02, San Francisco, January 2002.

[18] Pierreval H., Mebarki, N., Dynamic selection of dispatching rules for manufacturing system scheduling, International Journal of Production Research, vol. 35, nº, pp. 1575 $-1591,1997$.

[19] Pomerol, J. C., Barba-Romero, S. Choix multicritère dans l'entreprise : principe et pratique, ed. Hermes, 1993.

[20] Sim, S.K., Yeo, K.T., Lee, W.H., An expert neural network system for dynamic jobshop scheduling, International Journal of Production Research, vol. 32, n 8, pp. 1759 $1773,1994$.

[21] Smith, S.F., Knowledge-Base Production Management: Approaches, Results and Prospectives. Production Planning and Control, vol. 4, n³, pp. 350 - 380, 1992.

[22] Van Laarhooven, P. J. M., Aarts, E. H. L., and Lenstra, J. K., Job shop scheduling by simulated annealing, Operations Research, vol. 40, n 1 , pp. $113-125,1992$.

[23] Weigert, G., Werner, S., Hampel, D., Heinrich, H., and Sauer, W. Multi objective decision making Solutions for the optimization of manufacturing processes, $10^{\text {th }}$ International Conference FAIM'2000, Flexible Automation \& Intelligent Manufacturing Maryland, USA, Proceedings, pp. 487 - 496, 2000. 